\title{
Optimal Tests to Minimise Bleeding and Ischaemic Complications in Patients on Extracorporeal Membrane Oxygenation
}

\author{
Rahim Kanji ${ }^{1, *}$ Christophe Vandenbriele ${ }^{2,3, *}$ Deepa R. J. Arachchillage $e^{4,5}$ Susanna Price ${ }^{1,3, * *}$ \\ Diana Adrienne Gorog $1, * *($ C)
}

${ }^{1}$ Faculty of Medicine, National Heart and Lung Institute, Imperial

Address for correspondence Diana Adrienne Gorog, MD, PhD, FRCP, College, London, United Kingdom

2 Department of Cardiovascular Diseases, University Hospitals Leuven, Leuven, Belgium

${ }^{3}$ Intensive Care Unit, Royal Brompton Hospital, London, United Kingdom National Heart and Lung Institute, Imperial College, London, United Kingdom (e-mail: d.gorog@imperial.ac.uk).

${ }^{4}$ Haematology Department, Royal Brompton Hospital, London,

United Kingdom

${ }^{5}$ Centre for Haematology, Imperial College Healthcare NHS Trust \& Imperial College, London, United Kingdom

Thromb Haemost 2022;122:480-491.

\section{Abstract}

Keywords

- mechanical circulatory support

- thrombosis

- bleeding

- heparin

- anticoagulation

Patients supported with extracorporeal membrane oxygenation (ECMO) experience a very high frequency of bleeding and ischaemic complications, including stroke and systemic embolism. These patients require systemic anticoagulation, mainly with unfractionated heparin (UFH) to prevent clotting of the circuit and reduce the risk of arterial or venous thrombosis. Monitoring of UFH can be very challenging. While most centres routinely monitor the activated clotting time and activated partial thromboplastin time (aPTT) to assess UFH, measurement of anti-factor Xa (anti-Xa) level best correlates with heparin dose, and appears to be predictive of circuit thrombosis, although aPTT may be a better predictor of bleeding. Although monitoring of prothrombin time, platelet count and fibrinogen is routinely undertaken to assess haemostasis, there is no clear guidance available regarding the optimal test.

Additional tests, including antithrombin level and thromboelastography, can be used for risk stratification of patients to try and predict the risks of thrombosis and bleeding. Each has their specific role, strengths and limitations. Increased thrombin generation may have a role in predicting thrombosis. Acquired von Willebrand syndrome is frequent with ECMO, contributing to bleeding risk and can be detected by assessing the von Willebrand factor activity-to-antigen ratio, while the platelet function analyser can be used in urgent situations to detect this, with a high negative predictive value. Tests of platelet aggregation can aid in the prediction of bleeding.

To personalise management, a selection of complementary tests to collectively assess heparin-effect, coagulation, platelet function and platelet aggregation is proposed, to optimise clinical outcomes in these high-risk patients.
\end{abstract}

*Joint first authors.

**Joint senior authors.

received

January 29, 2021

accepted after revision

May 12, 2021

published online

May 13, 2021 (c) 2021. Thieme. All rights reserved.

Georg Thieme Verlag KG,

Rüdigerstraße 14 ,

70469 Stuttgart, Germany
DOI https://doi.org/

10.1055/a-1508-8230.

ISSN 0340-6245. 


\section{Introduction}

Both bleeding and ischaemic complications are frequent in patients on mechanical circulatory support (MCS). A metaanalysis of 1,866 adult patients with cardiogenic shock treated with extracorporeal membrane oxygenation (ECMO) reported major or significant bleeding in $40.8 \%$ of patients, lower limb ischaemia in $16.9 \%$ and stroke in $5.9 \%$ of patients. ${ }^{1}$ In patients receiving MCS, the artificial surface of the circuit activates the contact pathway of the coagulation cascade and, in combination with the inflammatory response, is an ongoing driver of thrombosis (-Fig. 1). At the same time, over-anticoagulation, low platelet count and/or acquired platelet dysfunction due to loss of platelet receptors through the MCS and acquired von Willebrand syndrome (AVWS) all contribute to an increased risk of bleeding. AVWS is frequent with ECMO and can greatly exacerbate bleeding.

Maintaining optimal haemostasis and preventing significant bleeding and thrombosis are key aspects of managing patients receiving ECMO. Anticoagulation is essential for leftsided support to prevent thrombotic complications and reduce the risk of systemic embolism, including stroke or limb ischaemia. ${ }^{2}$ This is usually achieved with intravenous unfractionated heparin (UFH), ${ }^{2}$ or the direct thrombin inhibitors bivalirudin or argatroban when UFH is contraindicated, such as in the case of allergy to UFH/heparin-induced thrombocytopenia (HIT), or in patients with severe acute antithrombin (AT) deficiency when treatment with AT concentrate is also an option. There are no randomised controlled trials to guide anticoagulation targets in patients on ECMO.

Maintaining optimal anticoagulation is challenging, as monitoring of UFH can be difficult in patients on $\mathrm{MCS}^{2}$ and the ideal strategy for monitoring remains unknown. In addition to assessing the effect of anticoagulation, it is also important to assess the overall haemostatic profile, to assess bleeding and thrombosis risk (-Fig. 1). Platelets play a major role in both primary and secondary haemostasis, and thrombocytopenia and/or impaired platelet function can contribute to the excess bleeding risk.

Depending on the centre, the method used for monitoring UFH varies, and may relate to the availability of the tests, as well as to clinician preference. The most commonly used tests include the activated clotting time (ACT), activated partial thromboplastin time (aPTT) and heparin levels as measured by anti-Xa level. Furthermore, to assess haemostasis, most centres offering ECMO routinely check prothrombin time (PT) and fibrinogen. ${ }^{2}$ Additionally, measurement of AT level and thromboelastography (TEG), as an assessment of global haemostasis, are reported to be used variably, or on an 'as-needed' basis. Newer tests are also available, but their role is often not well understood.

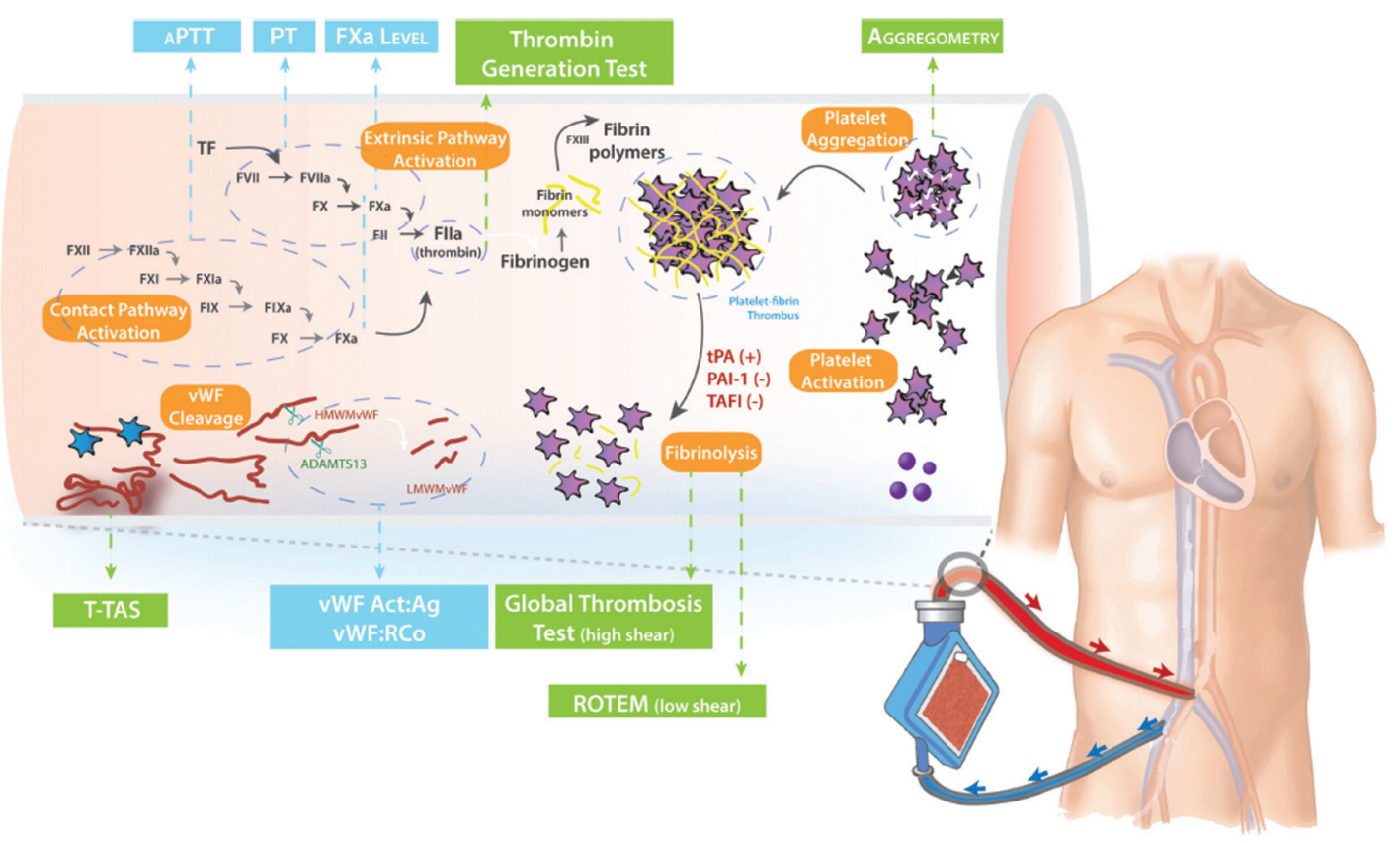

Fig. 1 Etiology of thrombosis and bleeding in patients on mechanical circulatory support and tests available to identify these risks. Contact of blood with the artificial circuit results in activation of coagulation, while platelet activation occurs due to the foreign surface and high shear flow conditions. Activation of coagulation and platelet aggregation result in platelet thrombus formation. Meanwhile, MCS particularly with continuous (as opposed to pulsatile) flow leads to cleavage of HMWM of vWF and development of AVWS, increasing the susceptibility to bleeding. Endogenous fibrinolysis, mediated predominantly by tissue-activated plasminogen activator (t-PA), plasminogen activator inhibitor-1 (PAI-1) and thrombin-activatable fibrinolysis inhibitor (TAFI), is responsible for prevention of lasting thrombotic occlusion and when enhanced can increase bleeding risk. Conventional tests to assess UFH shown in blue rectangles, more novel tests of haemostasis and fibrinolysis shown in green rectangles. AVWS, acquired von Willebrand syndrome; HMWM, high-molecular-weight multimer; MCS, mechanical circulatory support; UFH, unfractionated heparin; vWF, von Willebrand factor. 
Table 1 Tests available to monitor anticoagulation with unfractionated heparin

\begin{tabular}{|c|c|c|}
\hline Test & Advantages & Disadvantages \\
\hline ACT & $\begin{array}{l}\text { - Point-of-care test } \\
\text { - Rapid to perform } \\
\text { - Whole blood test }\end{array}$ & $\begin{array}{l}\text { - No clear validation in ECMO } \\
\text { - Affected by haemodilution, hypofibrinogenemia, } \\
\text { thrombocytopenia, liver failure, inflammation, lupus } \\
\text { anticoagulant and consumptive coagulopathy } \\
\text { - Used alone may lead to suboptimal anticoagulation } \\
\text { with ECMO }\end{array}$ \\
\hline aPTT & $\begin{array}{l}\text { - Point-of-care test available (not routinely) } \\
\text { - Best predictor of bleeding } \\
\text { - Relatively inexpensive }\end{array}$ & $\begin{array}{l}\text { - Often central laboratory: time delay } \\
\text { - High variation between reagents and laboratories } \\
\text { - Affected by many biologic factors, often disturbed in } \\
\text { critically ill patients } \\
\text { - Range based on anti-Xa }\end{array}$ \\
\hline Anti-Xa & $\begin{array}{l}\text { - Considered gold standard for assessing } \\
\text { heparin effect } \\
\text { - Good correlation with heparin dose } \\
\text { - Unaffected by presence of lupus anticoagulant, } \\
\text { liver disease or consumptive coagulopathy }\end{array}$ & $\begin{array}{l}\text { - Expensive } \\
\text { - Difficult to automate } \\
\text { - Not universally available } \\
\text { - Not point-of-care; central laboratory required: time delay }\end{array}$ \\
\hline
\end{tabular}

Abbreviations: ACT, activated clotting time; anti-Xa, anti-factor Xa; aPTT, activated partial thromboplastin time; ECMO, extracorporeal membrane oxygenation.

There are currently no guidelines regarding the optimal assessment of patients on ECMO. The aim of this review is to present available tests to assess the anticoagulant intensity of UFH and other haemostatic tests to predict bleeding and thrombosis, to provide guidance to practicing clinicians.

We performed a literature search using the MEDLINE/PubMed database, including articles published online from inception until January 2020. The search strategy is described in - Appendix A. As this is a narrative review, included articles were selected based on the authors' joint subjective determination of relevance to the overall theme of this article and the most pertinent articles informing clinical practice were referenced.

\section{Assessing Anticoagulant Effect}

The optimal anticoagulant monitoring strategy for UFH in patients receiving ECMO is undefined ( - Table 1). Overall, the dose of UFH best correlates with the anti-Xa level; however, whether levels relate to bleeding and thrombosis is not established in large studies. A study in paediatric ECMO patients reported poor correlation between UFH dose and either anti-Xa level $(\rho=0.1, p<0.0001)$ or aPTT $(\rho=0.26$, $p<0.0001$ ). Anti-Xa level and aPTT were weakly correlated ( $\rho=0.38, p<0.0001$ ) and neither was related to survival or haemorrhagic and thrombotic complications. ${ }^{3}$

\section{Activated Clotting Time}

In the critical care setting, UFH dose has previously been titrated to the measurement of the ACT, a widely available point-of-care assay. ${ }^{2}$ Performed on whole blood after addition of an activator (e.g., kaolin), it provides a rapid assessment of the adequacy of anticoagulation when high-dose UFH is used. However, it may not provide an accurate measure of UFH anticoagulant effect as a result of various confounding factors which are more marked in critically ill patients, including liver failure, haemodilution, thrombocytopenia, hypothermia, extreme fibrinogen levels, inflammation, lupus anticoagulant and consumptive coagulopathy. ${ }^{4}$ Although routinely used, its effectiveness to guide anticoagulation in this patient group has never been prospectively studied. Numerous reports indicate that monitoring UFH with ACT alone leads to suboptimal anticoagulation during MCS. In 46 adult patients on venoarterial (VA) or veno-venous (VV) ECMO, heparin dose correlated better with aPTT than $\mathrm{ACT}^{5}$ In paediatric patients receiving ECMO with comparable ACT levels, a $0.01 \mathrm{IU} / \mathrm{mL}$ decrease in anti-Xa level was shown to increase the risk of circuit thrombosis by $5 \%{ }^{6}$ In a retrospective analysis of 604 children on ECMO monitored with ACT in the range 180 to 220 seconds, there was an improved survival with increased heparin dose and ACT did not correlate with heparin dose, implying that ACT may be too insensitive to guide systemic anticoagulation. $^{7}$

\section{Activated Partial Thromboplastin Time}

Although aPTT is a commonly-used, inexpensive and readily available test, it may not provide an accurate measure of the amount of UFH present because of various confounding factors including both pre-analytical and analytical variables. The aPTT is measured by adding a surface activator and calcium to citrated plasma. Both laboratory and point-ofcare aPTT tests are available. In the intensive care setting, aPTT correlates better with heparin concentration than ACT, although the optimal aPTT target range and the correlation between aPTT and bleeding risk in patients on ECMO remains unclear., ${ }^{5,8}$ Both laboratory and point-of-care aPTT tests correlate poorly with heparin anti-Xa levels. Although several studies have shown that elevated aPTT level is a better predictor of bleeding than anti-Xa level. In a study of 539 adult in-patients treated with UFH, discordant aPTT and anti-Xa values were found in $42 \%$ of paired samples. ${ }^{9}$ In these patients, a disproportionately higher aPTT was associated with increased bleeding ( 9 vs. $3 \%, p=0.03$ ) and 30-day mortality ( 14 vs. $5 \%, p=0.02$ ). In a prospective study of 202 
paediatric patients undergoing cardiac surgery, an elevated aPTT, but not anti-Xa level, was associated with bleeding. ${ }^{10}$ In a retrospective analysis of 149 adult patients on ECMO (111 VA and $38 \mathrm{VV}$ ), higher aPTT levels were independently associated with bleeding. ${ }^{11}$ These findings may be a reflection of intrinsic coagulation abnormalities rather than the direct effect of UFH.

There is significant variability in aPTT readings between laboratories, different instruments and even between reagents. The American College of Chest Physicians recommends the determination of aPTT goal ranges for individual institutions based on heparin (anti-Xa) levels, indicating that heparin level monitoring may be a superior approach. ${ }^{12}$

\section{Anti-Factor Xa Level}

The anti-Xa assay evaluates the dose of heparin available in the circulation and may be considered a surrogate measurement of the overall anticoagulant activity of UFH. The assay measures the ability of a patient's plasma to inhibit exogenous added factor Xa (FXa), hydrolysing its synthetic substrate (chromogenic assay) or the anti-Xa activity is measured by performing an aPTT-based FX assay on each dilution without exogenous FXa (clotting-based assay).

Heparin anti-Xa assay that does not contain exogenous AT may give a more accurate estimation of anticoagulation in plasma, thus reflecting the in vivo UFH effect for the specific patient. ${ }^{13}$ However, it may not detect all the heparin present in that sample. Some chromogenic anti-Xa assays involve the addition of exogenous AT, which may increase the measured anti-Xa activity, and others that do not add exogenous AT, and the latter maybe considered a more physiological assessment. ${ }^{14}$ However, although anti-Xa assays with added AT trend higher than those without, a recent paper found a good correlation between the two types of anti-Xa platforms in paediatric patients treated with low-molecular-weight heparin even when the AT activity was $<70 \%{ }^{15}$

However, while the anti-Xa level is a direct measure of heparin effect, it does not reflect the overall haemostatic status, which may depend on the amount of thrombin/AT and pro-coagulant drivers. It is an expensive test and not widely available. However, unlike aPTT, anti-Xa levels are not affected by the presence of lupus anticoagulant, liver disease or consumptive coagulopathy.

Small case series in paediatric patients suggest that antiXa levels correlate better with heparin dosing, and probably the overall anticoagulation state during ECMO, than does ACT or a combination of ACT and aPTT. ${ }^{16-18}$ Small retrospective studies demonstrated that the anti-Xa level was predictive of circuit thrombosis in paediatric ${ }^{6}$ and adult patients ${ }^{19}$ on ECMO, where for every unit decrease in anti-Xa level, there was a sevenfold increase in deep venous thrombosis. ${ }^{19}$ However, some studies indicate that for the assessment of bleeding risk, aPTT is superior to anti-Xa for monitoring UFH in paediatric patients undergoing cardiac surgery, ${ }^{10}$ and in adult patients receiving ECMO. ${ }^{20}$ This could be due the fact that aPTT is reflective of both heparin and the other intrinsic coagulation factors, whereas anti-Xa levels indicate only the heparin levels present. In a retrospective single-centre study of 34 adults on ECMO, in whom heparin was monitored by anti-Xa and/or aPTT, some $15 \%$ of patients experienced a major thrombotic event and $27 \%$ experienced a haemorrhagic event. ${ }^{20}$ The anti-Xa assay better correlated with weight-based heparin dose than aPTT. Low anti-Xa values were predictive of thrombosis, whereas high aPTT values were predictive of bleeding. In a prospective study of 202 paediatric patients treated with UFH after cardiac surgery, in those who experienced bleeding events, the highest aPTT and corresponding anti-Xa for the 24 hours before bleeding events were used to assess the predictive value of these tests for bleeding. While there was a moderate correlation between aPTT and anti-Xa, aPTT $>150$ seconds was significantly related to bleeding (odds ratio: 1.71 per 10-second increase in aPTT; 95\% confidence interval: 1.21-2.42; $p=0.003$ ), whereas anti-Xa was not associated with bleeding. ${ }^{10}$ Therefore, a combination of anti-Xa and aPTT monitoring approach to detect thrombotic and bleeding risk may be preferable to either alone.

\section{Antithrombin III Level and Anticoagulant Effect of UFH} AT is essential as a cofactor for UFH to exert its anticoagulant effect. AT deficiency may result in inadequate anticoagulation with UFH at usual doses. Acquired AT deficiency is common in acutely unwell patients due to either decreased production or increased consumption, especially with MCS. While a consensus definition is lacking, 'heparin resistance' (HR) refers to the requirement of very high doses of heparin usually in excess of 35,000 units within a 24-hour period to maintain the anti-Xa level or aPTT within the therapeutic range. ${ }^{21}$ The prevalence of HR with extracorporeal circuits was originally derived from large case series of patients undergoing cardiopulmonary bypass, including those supported with intra-aortic balloon pumps. In these studies, the prevalence of HR was around $20 \%,{ }^{22-24}$ depending on the definition of HR used, and was associated with adverse outcomes. ${ }^{25}$ A large retrospective study in paediatric patients on ECMO showed that subtherapeutic aPTT was associated with increased mortality and increased heparin dosing conferred a survival advantage. ${ }^{7}$ However, a more recent small study of 69 neonatal and paediatric ECMO patients showed that neither dose of UFH nor time at therapeutic anti-Xa or aPTT levels affected the risk of bleeding or survival. ${ }^{3}$ The prevalence of HR is reported in only two studies of patients on ECMO. In one study of 67 adult patients on VA or VV ECMO, half the patients had HR, ${ }^{21}$ and there was no apparent relationship between HR and thrombotic or bleeding complications. ${ }^{21}$ A recent study of 81 adult patients with COVID-19 receiving VV ECMO and renal replacement therapy indicated that all patients met the criteria for HR. ${ }^{26}$ The Extracorporeal Life Support Organization (ELSO) recommends maintaining AT levels within the normal range (80-120\% of control) during ECMO, ${ }^{13}$ although AT assays are not readily available in all hospitals. Low AT can be treated by giving fresh frozen plasma, cryoprecipitate, human plasma-derived AT or recombinant AT concentrate, although the latter is expensive. Although not formally evaluated in patients supported with ECMO, AT concentrate 
is preferable to cryoprecipitate or fresh frozen plasma, since the latter carries the potential risk of transfusion-transmitted infections and contains only a small concentration of AT unit per volume of plasma (fresh frozen plasma contains only $1 \mathrm{U} / \mathrm{mL}$ of $\mathrm{AT}$ ), necessitating large volumes to normalise AT levels, which could lead to volume overload or transfusionrelated acute lung injury. ${ }^{27}$

Low AT may be a particular problem in neonates, who have a physiologic deficiency of AT, with levels as low as $40 \%$ of the adult range and this can be exacerbated by sepsis and MCS. In adults, there are no studies showing the clear benefit of closely monitoring and maintaining the AT level within the recommended range, with recent data suggesting that AT supplementation may not decrease heparin requirements, not diminish the incidence of bleeding and/or thrombosis in adult patients on ECMO. ${ }^{28}$

\section{Tests to Assess the Haemostatic State of Patients on MCS}

\section{Thromboelastography and Thromboelastometry}

Both TEG and rotational thromboelastometry (ROTEM) provide information on the characteristics of clot produced ex vivo under low shear conditions. As the clot starts to form, changes in impedance reflect the dynamics of clot formation, viscoelastic clot strength and clot lysis. Both are point-ofcare tests and utilise either native blood, which must be tested immediately, or citrated whole blood, which is stable for up to 2 hours at room temperature and requires recalcification prior to analysis.

Drawbacks include the necessity for staff training and inter-operator variability with TEG, which unlike ROTEM, is not automated, and it can take up to 2 hours to obtain results. Use of activators of coagulation such as tissue factor can accelerate the test producing results in 15 minutes, although this compromises the physiological relevance of the test.

Both TEG and ROTEM are used in cardiopulmonary bypass to assess haemostasis and guide blood transfusion requirements. Hyperfibrinolysis based on the manufacturers' definitions of maximum lysis $>15 \%$ in the ROTEM or Lysis $30>8 \%$ in the TEG, a measurement derived from the reduction of clot firmness over time, has been shown to be associated with increased bleeding. However, data supporting their usefulness in the setting of ECMO are less robust. A retrospective evaluation of 27 paediatric patients on MCS, predominantly VA ECMO, showed a weak correlation between TEG variables and aPTT, and between TEG and ACT, with a stronger correlation between TEG (maximum amplitude, MA) and platelet count. ${ }^{29} \mathrm{~A}$ retrospective analysis of prospective data to assess UFH effect in adults on VV or VA ECMO showed a weak correlation between ROTEM clotting time and aPTT, with no agreement between the directional changes of aPTT and ROTEM clotting time results on successive days. ${ }^{30}$

Small studies showed that a reduced maximum clot firmness (MCF) was predictive of bleeding in adult patients on ECMO, while a low MCF was observed in only $17 \%$ of nonbleeders. ${ }^{31}$ In 23 patients initiated on ECMO and 24 on ventricular assist (Berlin Heart Excor), MCF was predictive of bleeding and 30-day mortality. ${ }^{32}$ In 57 adult patients on VV ECMO, clotting time, but not MCF, correlated with severity of bleeding. ${ }^{33}$ In terms of transfusion requirements, a prospective trial of 42 adult patients on VV ECMO randomised to TEG-guided or aPTT-guided anticoagulation protocols showed no significant difference in bleeding or thrombotic events. ${ }^{34}$ Furthermore, a study in 57 adult patients showed that VV ECMO leads to a continuous increase in clotting time and a decrease in MCF the longer the ECMO lasts, but addition of ROTEM to aPTT or fibrinogen measurement did not aid the prediction of bleeding. ${ }^{33}$ The role of these viscoelastic tests in assessing thrombosis is even weaker. In a retrospective chart review of 30 paediatric patients on VA or VV ECMO, a TEG Rtime greater than 17.85 minutes and anti-Xa activity greater than $0.25 \mathrm{IU} / \mathrm{mL}$ were independent predictors of thrombosis. ${ }^{35}$ However, a meta-analysis including nine studies of paediatric patients on ECMO concluded that none of the measures of heparin anticoagulation, including TEG-R, correlated with either bleeding or thrombotic episodes. ${ }^{36}$

Thus, there are limited data indicating a role for TEG/ROTEM in predicting bleeding and possibly to guide anticoagulation and transfusion in patients on MCS, but not in predicting thrombotic complications.

\section{Thrombin Generation}

Thrombin (human activated coagulation factor II) is one of the key players in the haemostatic system. Activation of both the extrinsic (tissue factor) and intrinsic (contact) coagulation pathways results in conversion of prothrombin to thrombin via FXa, resulting in fibrin formation. Whereas most tests focus on a particular part of the thrombotic process (e.g., d-dimer levels on fibrin degradation, aPTT on the intrinsic pathway), thrombin generation (TG) provides a more global assessment of coagulation which assesses the capacity of blood to generate thrombin after stimulating the coagulation pathway with exogenous tissue factor. Specifically, lag time (time to minimum thrombin formation), peak height (maximum thrombin concentration) and endogenous thrombin potential (ETP), which is the total amount of thrombin generated (area under the curve [AUC]), are reported. The greater the TG, the greater the potential for thrombosis and the less for bleeding. TG can be assessed in platelet-poor, platelet-free or platelet-rich plasma, where the latter enables the investigation of interaction between platelets and coagulation factors, which closely mimics in vivo conditions. An advantage of the TG assay is that it measures the full potential of the plasma sample to generate thrombin, whereas conventional coagulation tests (PT, aPTT) terminate with fibrin clot formation when $95 \%$ of thrombin is yet to be formed. However, TG is an expensive specialised laboratory test, not rapidly available in the acute setting and more widely used in research. Automated tests such as the Thrombinoscope version of the calibrated automated thrombogram are becoming available, but data are scarce.

TG is mainly used to asses congenital or acquired bleeding disorders related to haemostasis, or to assess antithrombotic treatment. Outwith the setting of ECMO, several retrospective and prospective observational studies have shown a strong 
correlation between high ETP and the occurrence of venous thrombosis, ${ }^{37-39}$ although data on ECMO patients are sparse. In a small study, peak TG and ETP were significantly higher in samples from 20 patients on ECMO compared with control plasma. ${ }^{40}$ However, although half the cohort experienced a pulmonary embolism, there were no significant differences in lag time, peak TG, or ETP between patients with and without pulmonary embolism.

Although there is a strong correlation between increased TG and the occurrence of venous thrombosis, ${ }^{41}$ a relationship with arterial thrombosis has not been established. Reduced TG in patients on cardiopulmonary bypass is related to perioperative bleeding. ${ }^{42} \mathrm{~A}$ small study assessing TG in adult patients on VA ECMO showed an increased peak TG, and an increased ETP compared with controls, indicative of a procoagulant profile. ${ }^{40}$ Tests of TG may therefore have a role in the prediction of both bleeding and thrombosis, but convincing clinical data in the setting of ECMO are lacking.

\section{D-dimer}

D-dimer is a fibrin degradation product, produced as a result of fibrin formation and degradation. Although marked elevations may indicate the presence of venous thromboembolism or disseminated intravascular coagulation, D-dimer is a non-specific marker that is elevated in other conditions including sepsis. Individual D-dimer levels are not helpful in patients on ECMO, but daily measurement is recommended since an increase in D-dimer in the absence of other explaining pathology during ECMO therapy may reflect coagulation activity within the membrane oxygenator, ${ }^{43}$ and decrease after circuit exchange. ${ }^{44}$ Rising levels of Ddimer are usually considered in the context of other markers, such as a rising plasma-free haemoglobin and falling fibrinogen, which together should prompt the need for urgent consideration of circuit change.

\section{Assessment of Platelet Count and Platelet Function}

Regular assessment of platelet count is essential, with vigilance for the occurrence of HIT, which occurs in some 4 to $7 \%$ of ECMO patients. ${ }^{45} \mathrm{~A}$ low platelet count or a fall in platelet count should prompt consideration of HIT and various scores are available to gauge the likelihood of this. ${ }^{46}$ In individuals with suspected HIT, heparin should be discontinued and replaced with a non-heparin anticoagulant, while awaiting laboratory confirmation of HIT, which is initially assessed with enzymelinked immunosorbent assay for antiplatelet factor 4 antibodies.

\section{Light Transmission Aggregometry and Multiple Electrode} Aggregometry

Light transmission aggregometry (LTA) is considered the gold standard to assess platelet aggregation. However, it involves multiple steps and specialist laboratory expertise. It utilises anticoagulated platelet-rich plasma to which various agonists are added to stimulate platelet aggregation, including collagen, thrombin and thromboxane A2. Multiple electrode aggregometry (MEA) is a later derivative of impedance aggregometry, which employs anticoagulated whole blood, can be used as a point-of-care technique and has been shown to predict bleeding in patients undergoing cardiopulmonary bypass. ${ }^{47}$

In an experimental artificial circuit perfused with human blood, initiation of mechanical circulation was associated with reduction in platelet count and platelet aggregation as measured using LTA and MEA. ${ }^{48}$

There are small studies showing reduced platelet aggregation in adult patients on ECMO ${ }^{49}$ However, assessment of platelet function in patients on ECMO can be challenging, since it can be compounded by the presence of thrombocytopenia, present in some $25 \%$ of VV and $23 \%$ of VA ECMO patients, which begins within 2 to 3 days and is not related to duration of ECMO. ${ }^{50} \mathrm{~A}$ recent meta-analysis of 21 studies evaluating patients on ECMO revealed impaired platelet function, predominantly impaired platelet aggregation, in several studies, and a few studies also demonstrated reduced platelet adhesion and activation, including granule secretion. ${ }^{50} \mathrm{~A}$ meta-analysis of three paediatric ECMO studies demonstrated reduced platelet function as assessed using platelet aggregometry, flow cytometry and TEG-platelet mapping, which in two studies was irreversible by platelet transfusion. ${ }^{51}$ However, this should be interpreted with caution since another recent small study of 33 adults on VA or VV ECMO showed that both platelet count and platelet aggregation were reduced on ECMO, but when aggregation was assessed relative to platelet count, this did not differ from that of healthy controls. ${ }^{52}$ Furthermore, mild haemolysis is common in patients on ECMO, with severe haemolysis in 2 to $20 \%$ of patients, which can further compound platelet function analysis performed using whole blood. ${ }^{53}$

If the LTA or MEA shows significant platelet dysfunction in the presence of major bleeding, antiplatelet should be withheld and consideration should be given to platelet transfusion, even in the presence of normal platelet count. In the case of minor bleeding, the risk of withholding antiplatelet treatment should be balanced against the risk of bleeding. If the bleeding risk exceeds ischaemic risk, antiplatelet therapy should be withheld until the bleeding settles. If the thrombosis risk is high, antiplatelet therapy should be continued and tranexamic acid administered for 24 to 48 hours.

\section{Role of von Willebrand Factor in Haemostasis}

von Willebrand Factor (vWF) is a high-molecular-weight multimer (HMWM), synthesised and released by endothelial cells. It plays a crucial role in platelet-subendothelium adhesion and platelet-platelet interaction, and functions as a carrier protein for coagulation factor VIII. vWF alters from an irregularly coiled to an extended thread-like state in the transition from shear to elongational flow at sites of haemostasis and thrombosis. Transition from pulsatile to continuous blood flow, as seen in patients with continuous-flow MCS, leads to cleavage of large vWF multimers into monomers by the matrix metalloproteinase ADAMTS-13. Once cleaved, lower-molecular-weight multimers lose their affinity for binding platelets, increasing the susceptibility to bleeding. This imbalance between degradation induced by shear stress and the endothelial release of new vWF triggered by pulsatile flow during MCS results in overall reduction in HMWM, leading to 
device-induced AVWS, characterised by bleeding mainly from the mucosal surfaces, including gastrointestinal bleeding. ${ }^{54}$ The high shear in MCS appears to cause shedding of the platelet glycoproteins Ib (GPIb)/IX and VI, reducing vWF binding on the platelet surface and increasing the propensity for bleeding. ${ }^{55}$

The diagnosis of AVWS is made by detecting abnormally low vWF activity in relation to vWF antigen (vWF:Ag) levels, reflected in a deficiency of HMWM on gel electrophoresis. Although the latter is the gold standard for diagnosing AVWS, this is generally not readily available, and most centres use the ristocetin cofactor assay ( $\mathrm{vWF:RCo}$ ) to assess vWF activity in relation to vWF:Ag. The normal vWF:RCo/vWF:Ag ratio is $>0.7$, and a ratio of $<0.6-0.7$ indicates a loss of HMWM and AVWS. Although assessment of vWF:RCo with platelet aggregometry has been regarded as the 'gold standard', this demonstrates high variability and relatively low sensitivity for AVWS. Automated tests for vWF:RCo using turbidimetry or chemiluminescence have greatly improved precision, with markedly reduced coefficients of variation. ${ }^{56}$ Other automated tests include the vWF:GPIbR in which platelets are replaced with latex beads coated with recombinant wild-type GPIb and the vWF:Ab test which uses polystyrene beads coated with monoclonal antibody directed against the GPIb-binding A1 domain of vWF. ${ }^{56}$ These tests may eventually replace the vWF:RCo performed by aggregometry in many laboratories. To date, there are no point-ofcare tests specifically for AVWS especially in the setting of MCS, but adaptations of the platelet function analyser and viscoelastic tests may hold promise (vide infra).

There is evidence that use of continuous-flow mechanical support devices and ECMO leads to substantial reduction in HMWM of vWF and AVWS-related gastrointestinal bleeding. ${ }^{54,57}$ Indeed, the permanent high shear stress and continuous blood flow not only induce proteolysis of HMWMs, but also result in platelet inhibition and reorganisation of endothelial cells, particularly in the blood vessels of the gastrointestinal tract, leading to gastrointestinal dysplasia and subsequently bleeding. ${ }^{57,58}$ ECMO is generally associated with loss of HMWMs within 24 hours of treatment, with rapid reversal after withdrawal of MCS. ${ }^{57}$ In more than 200 patients on long-term pulsatile and continuous-flow MCS, development of AVWS, vWF:Ag and vWF:RCo levels was predictive of bleeding. ${ }^{59}$ The incidence of bleeding is significantly higher with non-pulsatile, continuous-flow assist devices than that with pulsatile-flow devices. Even using the same non-pulsatile device (ex vivo ECMO circuit), lowering the flow rate may enhance the loss of HMWMs. ${ }^{60}$ Importantly, patients supported with the latest generation left ventricular assist devices (lower pump speed, different textured interior surfaces, physiological pulsatile flow) had more intact HMWMs and higher vWF activity compared with patients treated with older devices. ${ }^{61}$

\section{Possible Future Monitoring Approaches}

\section{Point-of-Care Tests to Assess AVWS}

Although not evaluated in the setting of MCS, the PFA-100 appears to be a good screening test for von Willebrand disease (vWD), with high sensitivity. A study in 41 paediatric outpatients showed that prolonged closure time with the collagen/adenosine diphosphate (ADP) cartridge had an overall sensitivity of $90 \%$ to detect vWD. ${ }^{62}$ Specifically, all patients with type 2 or 3 vWD had prolonged PFA-100 closure times with both cartridge types, whereas amongst those with type 1 vWD, $83 \%$ had prolonged closure times with the collagen/ADP cartridge and $79 \%$ with collagen/epinephrine. A recent review confirms that the PFA-100/200 can be used in urgent situations to exclude the presence of AVWS with high sensitivity if closure time is normal, with a negative predictive value (NPV) $>99 \%,{ }^{63}$ although validation specifically in the setting of ECMO is lacking. However, a prolonged closure time in either the collagen/epinephrine or the collagen/ADP channel has very low positive predictive value (PPV) for AVWS, since such closure time prolongation is also seen with thrombocytopenia, low haematocrit and acquired platelet function defects. ${ }^{63}$

The latest version of MEA incorporates a RISTOtest, containing ristocetin for the quantitative determination of vWFand GPIb-dependent platelet aggregation, but is available for research use only.

The performance of a modified TEG assay to screen for vWD was evaluated in 328 patients, assessing the ratio of clot strength (MA) with and without pre-incubation of blood with ristocetin. A decrease in MA with a cut-off of $25 \%$ for the AUC ratio resulted in a sensitivity of 53 to $100 \%$ for different types of vWD. ${ }^{64}$ The same group subsequently compared the performance of TEG and ROTEM in 100 patients with vWD and 89 healthy controls. ${ }^{65}$ Prolonged TEG R-time had a PPV of 0.84 and a NPV of 0.68 respectively, while the clotting index (CI) had a PPV of 1.00 and an NPV of 0.60 . Both R-time and CI had a high specificity and accurately discriminated VWD patients from healthy controls, with an AUC of 0.85 and 0.99 , respectively. On the other hand, ROTEM parameters could not differentiate patients with vWD from healthy controls. ${ }^{65}$ However, a recent modification of the ROTEM assay with preincubation of the blood sample with ristocetin and commercially available vWF has shown promise in identifying vWD in a small study of 27 out-patients, ${ }^{66}$ but there has been no further validation.

\section{Quantification of vWF Cleavage}

A relatively recent development is a mass spectrometry method to quantify vWF cleavage as the ratio of the ADAMTS-13-cleaved peptide MVTGNPASDEIK to the ILAGPAGDSNVVK peptide. While this has not been evaluated in ECMO patients, increased vWF cleavage was detected in samples from patients on left ventricular assist devices who had developed bleeding. ${ }^{67}$ However, this requires special laboratory expertise and is time-consuming, such that it may not be practical for routine clinical use.

\section{Global Thrombosis Test}

This automated point-of-care test assesses the time taken to form an occlusive thrombus under high shear stress (occlusion time), and in the subsequent phase of the test, measures the time taken for spontaneous restart of flow, as a measure of endogenous fibrinolysis (lysis time). It assesses native, 
non-anticoagulated whole blood and is a global test of thrombosis. It has been shown to identify patients who are prothrombotic due to impaired endogenous fibrinolysis, in the setting of acute coronary syndromes. ${ }^{68,69}$ As it provides a global assessment of thrombotic status, including platelet reactivity and coagulation in the setting of high shear, it would seem an ideal point-of-care test in patients on ECMO. It could be used to assess the risk of thrombosis (short occlusion time and/or long lysis time) and bleeding (long occlusion time and/or short lysis), but studies to date have not been performed in this patient group.

\section{Total Thrombus-Formation Analysis System}

This is a relatively novel point-of-care flow chamber system which employs two microchips; the platelet (PL) chip AUC assesses platelet function and primary haemostasis, while the atheroma (AR) chip assesses overall haemostatic ability. It has been shown to be of use in monitoring the effects of antiplatelet therapy and the severity of vWD type 1, with small AUC predictive of peri-procedural bleeding in patients undergoing stenting. ${ }^{70}$ In four patients on continuous flow left ventricular assist devices, HMWM vWF and Total Thrombus-formation Analysis System (T-TAS) PL24-AUC10 and AR10-AUC30 were significantly reduced compared with anticoagulated control patients. ${ }^{70}$ Future studies are required to see whether it could be used to detect AVWS in patients on ECMO.

\section{Growth Differentiation Factor-15}

Growth differentiation factor-15 (GDF-15), a member of the transforming growth factor- $\beta$ superfamily, is upregulated in many disease states including cardiovascular disease, has been shown to reduce ADP-induced platelet aggregation in a dose-dependent manner and is a marker of bleeding. In large studies in patients on dual antiplatelet therapy or oral anticoagulation, elevated GDF-15 levels were independently associated with major bleeding during follow-up. ${ }^{71,72} \mathrm{Circu}-$ lating GDF-15 level has been shown to be elevated in patients with heart failure prior to left ventricular assist device implantation, and to fall significantly after 1 month of mechanical unloading, ${ }^{73}$ but there are no studies assessing its utility to predict bleeding in patients on ECMO.

\section{Possible Novel Anticoagulant Approaches}

Exposure of blood to artificial surfaces potentiates thrombosis through contact activation, namely the activation of factor XII (FXII), XI (FXI) and plasma prekallikrein. Targeted inhibition of FXIa or FXIIa may reduce thrombotic risk associated with contact activation, while leaving haemostasis largely intact, $^{74}$ an avenue that seems particularly attractive in patients on ECMO who require anticoagulation but are prone to excessive bleeding.

In rabbits connected to a paediatric ECMO circuit, a recombinant fully human FXIIa activity-neutralising antibody (3F7) dose-dependently reduced thrombus formation at arterial shear rates, without an increase in bleeding. ${ }^{75}$ In primates, monoclonal antibodies against FXII reduced thrombosis in collagen-coated arteriovenous grafts. ${ }^{76}$ In rabbits, antisense oligonucleotides (ASOs) inhibiting the production of FXII or FXI prolonged the time to catheterinduced venous thrombosis, ${ }^{77}$ while a monoclonal immunoglobulin $G$ against FXIa was as effective as rivaroxaban in preventing venous thrombosis, without increasing cuticle bleeding. ${ }^{78}$ It remains to be established if FXII or FXI is the better target for thromboprophylaxis in patients supported by devices with artificial surfaces. While FXII inhibition may not increase the risk of bleeding, inhibition of FXI may produce a more potent antithrombotic effect. No clinical trials employing FXII inhibition in humans have been reported; however, there are several different approaches being developed to inhibit FXI, including ASOs, monoclonal antibodies, aptamers and small molecules. Small-molecule inhibitors can be delivered orally or parenterally, whereas ASOs, antibodies and aptamers require parenteral administration. Antibodies, aptamers and small molecules can achieve rapid FXI inhibition while ASOs can take 3 to 4 weeks to achieve therapeutic anticoagulation. In phase II trials in patients undergoing knee surgery, an ASO FXI inhibitor ${ }^{79}$ administered subcutaneously and an anti-FXIa antibody osocimab, ${ }^{80}$ given intravenously, have both been shown to be superior to enoxaparin for thromboprophylaxis without an increase in bleeding. ${ }^{79} \mathrm{~A}$ small-molecule oral FXIa inhibitor has commenced a phase IIb program (PACIFIC), enrolling more than 4,000 patients with atrial fibrillation, myocardial infarction, or stroke. Several ongoing phase II studies will assess the safety of FXI inhibition in patients with end-stage renal disease on haemodialysis. There are no studies in patients on MCS, although onset of effect, half-life and offset/reversibility will be key issues when considering such strategies in patients on ECMO.

\section{Guide to Haemostasis Assessment and Management}

We provide a summary and schematic for assessing thrombosis and bleeding risk in patients on ECMO (-Fig. 2), based on our experience combined with literature review. Anti-Xa level best correlates with heparin dose, and appears predictive of thrombosis, although aPTT may be a better predictor of bleeding. In the absence of data to guide optimal anticoagulation, the 2014 ELSO anticoagulation guideline states that the majority of ELSO centres that use the anti-Xa levels aim for a target of 0.3 to $0.7 \mathrm{IU} / \mathrm{mL}$, which is a very broad range. ${ }^{13}$ We use the lower end of the range, aiming for 0.2 to $0.5 \mathrm{IU} / \mathrm{mL}$ to reduce the risk of bleeding, with a lower target of 0.2 to $0.3 \mathrm{IU} / \mathrm{mL}$ for patients on VV ECMO, based on our experience ${ }^{45}$ and supported by a protocol in the EOLIA trial. ${ }^{81}$ HR should be considered in patients who are not achieving the desired anti-Xa level despite escalating doses of UFH. In such cases, the AT level should be checked and preferably maintained in the normal range, with consideration given to switching from UFH to a direct thrombin inhibitor such as argatroban. Patients with thrombocytopenia or those who are bleeding should be promptly investigated for HIT, with consideration of alternative anticoagulation. The patient should be assessed for AVWS 


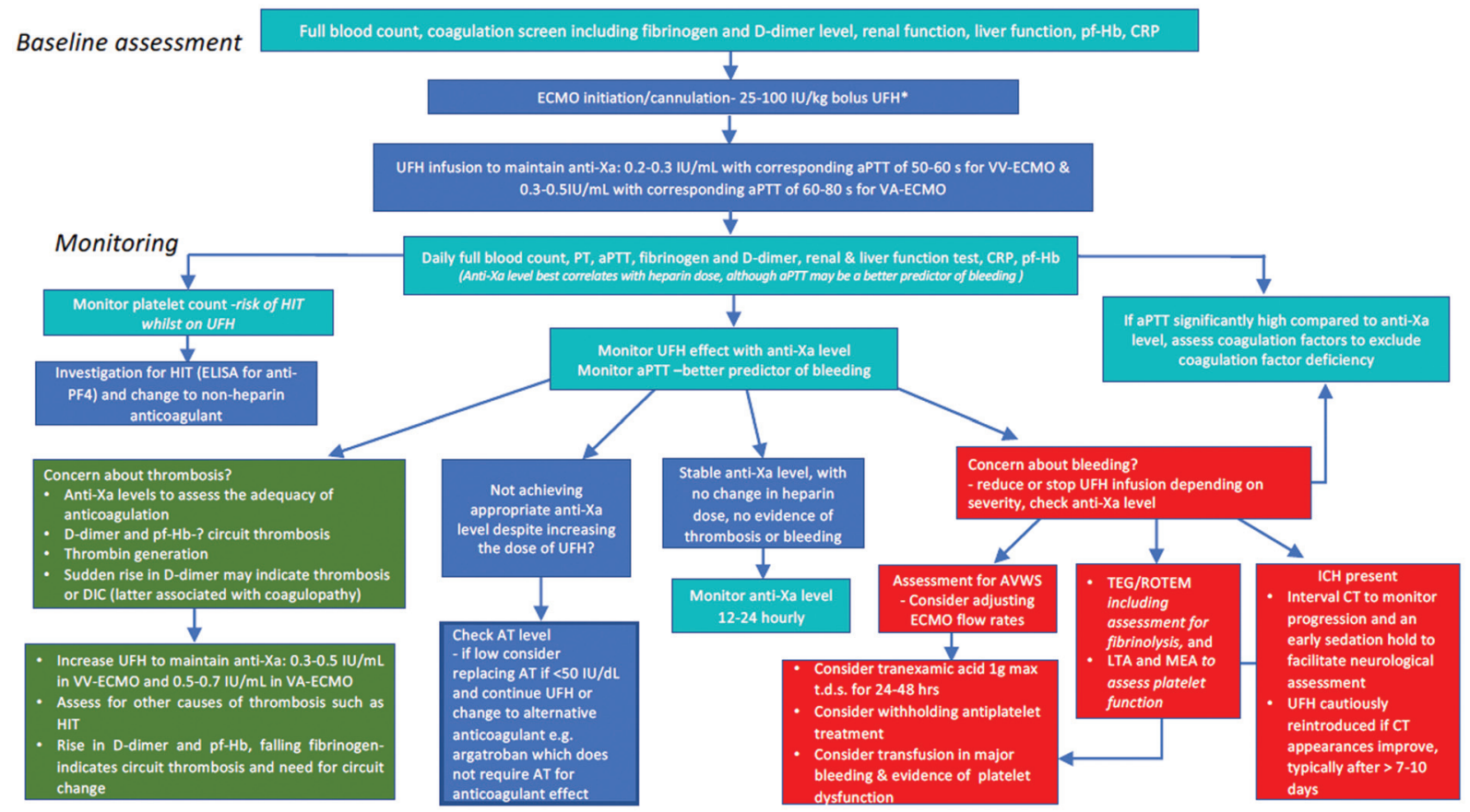

Fig. 2 Proposal for monitoring patients on mechanical circulatory support (MCS). Baseline assessment and monitoring of anticoagulation is required in all patients as shown. Patients on MCS are at risk of both thrombosis (green panels) and bleeding (red panels), requiring regular assessment (turquoise panels). To prevent bleeding while preventing thrombosis, we aim to keep heparin anti-Xa level in the target $0.2-0.5 \mathrm{IU} / \mathrm{mL}$, at the lower end of the therapeutic range. Patients on MCS are at risk of developing platelet dysfunction due to loss of platelet receptors and AVWS despite normal platelet count. Platelet function tests allow the identification of these patients and if the patient is bleeding despite normal coagulation assays and platelet count, assessment of platelet function can help reveal platelet dysfunction. In patients with recurrent thrombotic events, particularly arterial events, despite adequate anticoagulation, and normal or activated platelet response, the addition of antiplatelet treatment to anticoagulation can be considered. *Dose range is given to reflect the variation in practice. anti-PF4, antiplatelet factor 4 antibodies; aPTT, activated partial thromboplastin time; AT, antithrombin; AVWS, acquired von Willebrand syndrome; CRP, Creactive protein; CT, computed tomography; DIC, disseminated intravascular coagulation; ELISA, enzyme-linked immunosorbent assay; HIT, heparin-induced thrombocytopenia; ICH, intracranial haemorrhage; LTA, light transmittance aggregometry; MEA, multiple electrode aggregometry; pf-Hb, plasma-free haemoglobin; PT, prothrombin time; ROTEM, rotational thromboelastometry; t.d.s., ter die sumendum (3 times a day); TEG, thromboelastography; UFH, unfractionated heparin.

and ECMO flow rates should be adjusted to minimise AVWS whenever possible. Viscoelastic tests may be used to assess bleeding risk. Platelet aggregation should be assessed relative to platelet count, and in patients experiencing major bleeding with markedly impaired platelet aggregation on antiplatelet medication, consideration should be given to the risks and benefits of withholding antiplatelet medication as indicated by the clinical scenario (such as in the case of acute coronary syndrome, recent stent implantation in a major artery or last remaining conduit) in consultation with the wider clinical team(s). In addition, platelet transfusion should be considered, even in the presence of normal platelet count. If the ischaemic risk is high, antiplatelet therapy should be continued and tranexamic acid administered concomitantly for 1 to 2 days. In patients experiencing thrombosis, anti-Xa levels and possibly TG may be useful, where rising ETP may indicate a risk of venous thrombosis.

\section{Discussion}

There are no prospective randomised controlled studies assessing clinical outcomes in patients on ECMO receiving UFH which compare monitoring with aPTT versus heparin anti-Xa level, to guide recommendations for anticoagulant monitoring. Measurement of anti-Xa level best correlates with heparin dose, and appears predictive of thrombosis, although the aPTT level is a better predictor of bleeding. Platelets play a major role in both primary and secondary haemostasis. In addition to thrombocytopenia due to reduced bone marrow production, sepsis, pooling of platelets in the liver or spleen, and consumption of platelets through the MCS, there is also a high risk of platelet dysfunction which is directly related to the degree of shear and the duration of exposure to the artificial circuit. The high shear in MCS appears to cause VWF binding on the platelet surface and increasing the propensity for bleeding. AVWS, best detected by assessing the vWF:RCo ratio, is frequent, almost universal in patients on MCS, contributing to bleeding. Tests of platelet aggregation may be additionally used to predict bleeding. High shear stress also causes platelet activation and simultaneously increases the risk of thrombosis. Despite conventional monitoring of UFH, there is a high rate of thrombotic and bleeding complications with MCS. A selection of complementary tests to collectively assess heparin effect, coagulation, platelet function and platelet aggregation would be most useful for optimal early detection of bleeding and thrombosis risk, to personalise management and optimise outcomes in patients on MCS. 


\section{What is known about this topic?}

- Patients receiving extracorporeal membrane oxygenation (ECMO) have a very high frequency of bleeding and ischaemic complications that often jeopardise the patients' outcome and monitoring of antithrombotic therapy can be very challenging.

\section{What does this paper add?}

- We describe the selection of complementary tests which can collectively assess heparin effect, coagulation, platelet function and platelet aggregation, with supporting evidence in ECMO, to enable personalised management and optimise outcomes.

\section{Author Contributions}

D.A.G. and S.P. are responsible for the paper conception and design. C.V. and R.K. performed the literature search and data analysis and prepared the first draft. D.R.J.A. provided the haemostasis management algorithm. D.A.G., C.V., D.R.J.A. and S.P. all critically reviewed and revised the manuscript. All authors have approved the final version of the manuscript.

\section{Conflict of Interest}

None declared.

\section{References}

1 Cheng R, Hachamovitch R, Kittleson M, et al. Complications of extracorporeal membrane oxygenation for treatment of cardiogenic shock and cardiac arrest: a meta-analysis of 1,866 adult patients. Ann Thorac Surg 2014;97(02):610-616

2 Gorog DA, Price S, Sibbing D, et al. Antithrombotic therapy in patients with acute coronary syndrome complicated by cardiogenic shock or out-of-hospital cardiac arrest: a joint position paper from the European Society of Cardiology (ESC) Working Group on Thrombosis, in association with the Acute Cardiovascular Care Association (ACCA) and European Association of Percutaneous Cardiovascular Interventions (EAPCI). Eur Heart J Cardiovasc Pharmacother 2021;7(02):125-140

3 McMichael ABV, Hornik CP, Hupp SR, Gordon SE, Ozment CP. Correlation among antifactor $\mathrm{Xa}$, activated partial thromboplastin time, and heparin dose and association with pediatric extracorporeal membrane oxygenation complications. ASAIO J 2020; 66(03):307-313

4 Vandenbriele C, Vanassche T, Price S. Why we need safer anticoagulant strategies for patients on short-term percutaneous mechanical circulatory support. Intensive Care Med 2020;46(04):771-774

5 Atallah S, Liebl M, Fitousis K, Bostan F, Masud F. Evaluation of the activated clotting time and activated partial thromboplastin time for the monitoring of heparin in adult extracorporeal membrane oxygenation patients. Perfusion 2014;29(05):456-461

6 Irby K, Swearingen C, Byrnes J, Bryant J, Prodhan P, Fiser R. Unfractionated heparin activity measured by anti-factor Xa levels is associated with the need for extracorporeal membrane oxy- genation circuit/membrane oxygenator change: a retrospective pediatric study. Pediatr Crit Care Med 2014;15(04):e175-e182

7 Baird CW, Zurakowski D, Robinson B, et al. Anticoagulation and pediatric extracorporeal membrane oxygenation: impact of activated clotting time and heparin dose on survival. Ann Thorac Surg 2007;83(03):912-919

8 De Waele JJ, Van Cauwenberghe S, Hoste E, Benoit D, Colardyn F. The use of the activated clotting time for monitoring heparin therapy in critically ill patients. Intensive Care Med 2003;29(02): 325-328

9 Price EA, Jin J, Nguyen HM, Krishnan G, Bowen R, Zehnder JL. Discordant aPTT and anti-Xa values and outcomes in hospitalized patients treated with intravenous unfractionated heparin. Ann Pharmacother 2013;47(02):151-158

10 Oladunjoye OO, Sleeper LA, Nair AG, et al. Partial thromboplastin time is more predictive of bleeding than anti-Xa levels in heparinized pediatric patients after cardiac surgery. J Thorac Cardiovasc Surg 2018;156(01):332.e1-340.e1

11 Aubron C, DePuydt J, Belon F, et al. Predictive factors of bleeding events in adults undergoing extracorporeal membrane oxygenation. Ann Intensive Care 2016;6(01):97

12 Hirsh J, Raschke R. Heparin and low-molecular-weight heparin: the Seventh ACCP Conference on Antithrombotic and Thrombolytic Therapy. Chest 2004;126(3, Suppl):188S-203S

13 Anticoagulation Guideline ELSO. 2014. Accessed April 20, 2021 at: https://www.elso.org/portals/0/files/elsoanticoagulationguideline8-2014-table-contents.pdf

14 Ignjatovic V, Summerhayes R, Gan A, et al. Monitoring unfractionated heparin (UFH) therapy: which anti-factor Xa assay is appropriate? Thromb Res 2007;120(03):347-351

15 Schaefer B, Hausfeld A, Martin M, et al. Impact of exogenous antithrombin on low molecular weight heparin anti-Xa activity assays in a pediatric and young adult leukemia and lymphoma cohort with variable antithrombin levels. Pediatr Blood Cancer 2020;67(11):e28654

16 Bembea MM, Schwartz JM, Shah N, et al. Anticoagulation monitoring during pediatric extracorporeal membrane oxygenation. ASAIO J 2013;59(01):63-68

17 Nankervis CA, Preston TJ, Dysart KC, et al. Assessing heparin dosing in neonates on venoarterial extracorporeal membrane oxygenation. ASAIO J 2007;53(01):111-114

18 Liveris A, Bello RA, Friedmann P, et al. Anti-factor Xa assay is a superior correlate of heparin dose than activated partial thromboplastin time or activated clotting time in pediatric extracorporeal membrane oxygenation*. Pediatr Crit Care Med 2014;15(02): e72-e79

19 Chu DC, Abu-Samra AG, Baird GL, et al. Quantitative measurement of heparin in comparison with conventional anticoagulation monitoring and the risk of thrombotic events in adults on extracorporeal membrane oxygenation. Intensive Care Med 2015;41(02):369-370

20 Arnouk S, Altshuler D, Lewis TC, et al. Evaluation of anti-Xa and activated partial thromboplastin time monitoring of heparin in adult patients receiving extracorporeal membrane oxygenation support. ASAIO J 2020;66(03):300-306

21 Raghunathan V, Liu P, Kohs TCL, et al. Heparin resistance is common in patients undergoing extracorporeal membrane oxygenation but is not associated with worse clinical outcomes. ASAIO J 2021;67(8):899-906

22 Staples MH, Dunton RF, Karlson KJ, Leonardi HK, Berger RL. Heparin resistance after preoperative heparin therapy or intraaortic balloon pumping. Ann Thorac Surg 1994;57(05): 1211-1216

23 Ranucci M, Isgrò G, Cazzaniga A, et al. Different patterns of heparin resistance: therapeutic implications. Perfusion 2002;17 (03):199-204 
24 Avidan MS, Levy JH, Scholz J, et al. A phase III, double-blind, placebo-controlled, multicenter study on the efficacy of recombinant human antithrombin in heparin-resistant patients scheduled to undergo cardiac surgery necessitating cardiopulmonary bypass. Anesthesiology 2005;102(02):276-284

25 Ranucci M, Frigiola A, Menicanti L, Ditta A, Boncilli A, Brozzi S. Postoperative antithrombin levels and outcome in cardiac operations. Crit Care Med 2005;33(02):355-360

26 Streng AS, Delnoij TSR, Mulder MMG, et al. Monitoring of unfractionated heparin in severe COVID-19: an observational study of patients on CRRT and ECMO. TH Open 2020;4(04):e365-e375

27 Chlebowski MM, Baltagi S, Carlson M, Levy JH, Spinella PC. Clinical controversies in anticoagulation monitoring and antithrombin supplementation for ECMO. Crit Care 2020;24(01):19

28 Panigada M, Cucino A, Spinelli E, et al. A randomized controlled trial of antithrombin supplementation during extracorporeal membrane oxygenation. Crit Care Med 2020;48(11):1636-1644

29 Alexander DC, Butt WW, Best JD, Donath SM, Monagle PT, Shekerdemian LS. Correlation of thromboelastography with standard tests of anticoagulation in paediatric patients receiving extracorporeal life support. Thromb Res 2010;125(05):387-392

30 Prakash S, Wiersema UF, Bihari S, Roxby D. Discordance between ROTEM ${ }^{\circledR}$ clotting time and conventional tests during unfractionated heparin-based anticoagulation in intensive care patients on extracorporeal membrane oxygenation. Anaesth Intensive Care 2016;44(01):85-92

31 Nair P, Hoechter DJ, Buscher H, et al. Prospective observational study of hemostatic alterations during adult extracorporeal membrane oxygenation (ECMO) using point-of-care thromboelastometry and platelet aggregometry. J Cardiothorac Vasc Anesth 2015;29(02):288-296

32 Laine A, Niemi T, Suojaranta-Ylinen R, et al. Decreased maximum clot firmness in rotational thromboelastometry (ROTEM $®$ ) is associated with bleeding during extracorporeal mechanical circulatory support. Perfusion 2016;31(08):625-633

33 Hellmann C, Schmutz A, Kalbhenn J. Bleeding during veno-venous ECMO cannot reliably be predicted by rotational thrombelastometry (ROTEM ${ }^{\mathrm{TM}}$ ). Perfusion 2018;33(04):289-296

34 Panigada M, Iapichino GE, Brioni M, et al. Thromboelastographybased anticoagulation management during extracorporeal membrane oxygenation: a safety and feasibility pilot study. Ann Intensive Care 2018;8(01):7

35 Henderson N, Sullivan JE, Myers J, et al. Use of thromboelastography to predict thrombotic complications in pediatric and neonatal extracorporeal membranous oxygenation. J Extra Corpor Technol 2018;50(03):149-154

36 Padhya DR, Prutsky GJ, Nemergut ME, et al. Routine laboratory measures of heparin anticoagulation for children on extracorporeal membrane oxygenation: Systematic review and meta-analysis. Thromb Res 2019;179:132-139

37 Besser M, Baglin C, Luddington R, van Hylckama Vlieg A, Baglin T. High rate of unprovoked recurrent venous thrombosis is associated with high thrombin-generating potential in a prospective cohort study. J Thromb Haemost 2008;6(10):1720-1725

38 van Hylckama Vlieg A, Christiansen SC, Luddington R, Cannegieter SC, Rosendaal FR, Baglin TP. Elevated endogenous thrombin potential is associated with an increased risk of a first deep venous thrombosis but not with the risk of recurrence. $\mathrm{Br} \mathrm{J}$ Haematol 2007;138(06):769-774

39 Wexels F, Dahl OE, Pripp AH, Seljeflot I. Thrombin generation in patients with suspected venous thromboembolism. Clin Appl Thromb Hemost 2017;23(05):416-421

40 Mazzeffi M, Strauss E, Meyer M, et al. Coagulation factor levels and underlying thrombin generation patterns in adult extracorporeal membrane oxygenation patients. Anesth Analg 2019;129(03): 659-666

41 Al Dieri R, de Laat B, Hemker HC. Thrombin generation: what have we learned? Blood Rev 2012;26(05):197-203
42 Bosch YPJ, Al Dieri R, ten Cate H, et al. Measurement of thrombin generation intra-operatively and its association with bleeding tendency after cardiac surgery. Thromb Res 2014;133(03):488-494

43 Lubnow M, Philipp A, Dornia C, et al. D-dimers as an early marker for oxygenator exchange in extracorporeal membrane oxygenation. J Crit Care 2014;29(03):473.e1-473.e5

44 Dornia C, Philipp A, Bauer S, et al. D-dimers are a predictor of clot volume inside membrane oxygenators during extracorporeal membrane oxygenation. Artif Organs 2015;39(09):782-787

45 Arachchillage DRJ, Laffan M, Khanna S, et al. Frequency of thrombocytopenia and heparin-induced thrombocytopenia in patients receiving extracorporeal membrane oxygenation compared with cardiopulmonary bypass and the limited sensitivity of pretest probability score. Crit Care Med 2020;48(05):e371-e379

46 Lo GK, Juhl D, Warkentin TE, Sigouin CS, Eichler P, Greinacher A. Evaluation of pretest clinical score (4 T's) for the diagnosis of heparin-induced thrombocytopenia in two clinical settings. J Thromb Haemost 2006;4(04):759-765

47 Ranucci M, Colella D, Baryshnikova E, Di Dedda USurgical and Clinical Outcome Research (SCORE) Group. Effect of preoperative P2Y12 and thrombin platelet receptor inhibition on bleeding after cardiac surgery. Br J Anaesth 2014;113(06):970-976

48 Van Poucke S, Stevens K, Kicken C, Simons A, Marcus A, Lancé M Platelet function during hypothermia in experimental mock circulation. Artif Organs 2016;40(03):288-293

49 Balle CM, Jeppesen AN, Christensen S, Hvas A-M. Platelet function during extracorporeal membrane oxygenation in adult patients: a systematic review. Front Cardiovasc Med 2018;5:157

50 Jiritano F, Serraino GF, Ten Cate $\mathrm{H}$, et al. Platelets and extracorporeal membrane oxygenation in adult patients: a systematic review and meta-analysis. Intensive Care Med 2020;46(06): 1154-1169

51 Yaw HP, Van Den Helm S, MacLaren G, Linden M, Monagle P, Ignjatovic V. Platelet phenotype and function in the setting of pediatric extracorporeal membrane oxygenation (ECMO): a systematic review. Front Cardiovasc Med 2019;6:137

52 Balle CM, Jeppesen AN, Christensen S, Hvas A-M. Platelet function during extracorporeal membrane oxygenation in adult patients. Front Cardiovasc Med 2019;6:114

53 Sniderman J, Monagle P, Annich GM, MacLaren G. Hematologic concerns in extracorporeal membrane oxygenation. Res Pract Thromb Haemost 2020;4(04):455-468

54 Uriel N, Pak S-W, Jorde UP, et al. Acquired von Willebrand syndrome after continuous-flow mechanical device support contributes to a high prevalence of bleeding during long-term support and at the time of transplantation. J Am Coll Cardiol 2010;56(15):1207-1213

55 Lukito P, Wong A, Jing J, et al. Mechanical circulatory support is associated with loss of platelet receptors glycoprotein Ib $\alpha$ and glycoprotein VI. J Thromb Haemost 2016;14(11):2253-2260

56 Higgins RA, Goodwin AJ. Automated assays for von Willebrand factor activity. Am J Hematol 2019;94(04):496-503

57 Kalbhenn J, Schlagenhauf A, Rosenfelder S, Schmutz A, Zieger B. Acquired von Willebrand syndrome and impaired platelet function during venovenous extracorporeal membrane oxygenation: rapid onset and fast recovery. J Heart Lung Transplant 2018;37 (08):985-991

58 Meyer AL, Malehsa D, Budde U, Bara C, Haverich A, Strueber M. Acquired von Willebrand syndrome in patients with a centrifugal or axial continuous flow left ventricular assist device. JACC Heart Fail 2014;2(02):141-145

59 Oezpeker C, Zittermann A, Baurichter D, et al. Changes in von Willebrand factor profile predicts clinical outcomes in patients on mechanical circulatory support. J Card Surg 2018;33(10): 693-702

$60 \mathrm{Ki}$ KK, Passmore MR, Chan $\mathrm{CHH}$, et al. Low flow rate alters haemostatic parameters in an ex-vivo extracorporeal membrane oxygenation circuit. Intensive Care Med Exp 2019;7(01):51 
61 Klaeske K, Dieterlen M-T, Scholz U, et al. Acquired von Willebrand factor deficiency is reduced in HeartMate 3 patients†. Eur J Cardiothorac Surg 2019;56(03):444-450

62 Dean JA, Blanchette VS, Carcao MD, et al. von Willebrand disease in a pediatric-based population-comparison of type 1 diagnostic criteria and use of the PFA-100 and a von Willebrand factor/collagen-binding assay. Thromb Haemost 2000;84(03): 401-409

63 Favaloro EJ. Utility of the platelet function analyser (PFA-100/200) for exclusion or detection of von Willebrand disease: a study 22 years in the making. Thromb Res 2020;188:17-24

64 Topf H-G, Weiss D, Lischetzki G, Strasser E, Rascher W, Rauh M. Evaluation of a modified thromboelastography assay for the screening of von Willebrand disease. Thromb Haemost 2011; 105(06):1091-1099

65 Schmidt DE, Majeed A, Bruzelius M, Odeberg J, Holmström M, Ågren A. A prospective diagnostic accuracy study evaluating rotational thromboelastometry and thromboelastography in 100 patients with von Willebrand disease. Haemophilia 2017;23(02):309-318

66 Topf H-G, Strasser ER, Breuer G, Rascher W, Rauh M, Fahlbusch FB. Closing the gap - detection of clinically relevant von Willebrand disease in emergency settings through an improved algorithm based on rotational thromboelastometry. BMC Anesthesiol 2019; 19(01):10

67 Zhou Y, Qin S, Hilton T, et al. Quantification of von Willebrand factor cleavage by ADAMTS-13 in patients supported by left ventricular assist devices. ASAIO J 2017;63(06):849-853

68 Farag M, Spinthakis N, Gue YX, et al. Impaired endogenous fibrinolysis in ST-segment elevation myocardial infarction patients undergoing primary percutaneous coronary intervention is a predictor of recurrent cardiovascular events: the RISK PPCI study. Eur Heart J 2019;40(03):295-305

69 Gorog DA, Lip GYH. Impaired spontaneous/endogenous fibrinolytic status as new cardiovascular risk factor?: JACC review topic of the week J Am Coll Cardiol 2019;74(10):1366-1375

70 Kaikita K, Hosokawa K, Dahlen JR, Tsujita K. Total thrombusformation analysis system (T-TAS): clinical application of quantitative analysis of thrombus formation in cardiovascular disease. Thromb Haemost 2019;119(10):1554-1562

71 Hagström E, James SK, Bertilsson M, et al; PLATO Investigators. Growth differentiation factor-15 level predicts major bleeding and cardiovascular events in patients with acute coronary syndromes: results from the PLATO study. Eur Heart J 2016;37(16): 1325-1333

72 Wallentin L, Hijazi Z, Andersson U, et al; ARISTOTLE Investigators. Growth differentiation factor 15 , a marker of oxidative stress and inflammation, for risk assessment in patients with atrial fibrillation: insights from the Apixaban for Reduction in Stroke and Other Thromboembolic Events in Atrial Fibrillation (ARISTOTLE) trial. Circulation 2014;130(21):1847-1858

73 Lok SI, Winkens B, Goldschmeding R, et al. Circulating growth differentiation factor-15 correlates with myocardial fibrosis in patients with non-ischaemic dilated cardiomyopathy and decreases rapidly after left ventricular assist device support. Eur J Heart Fail 2012;14(11):1249-1256

74 Mackman N, Bergmeier W, Stouffer GA, Weitz JI. Therapeutic strategies for thrombosis: new targets and approaches. Nat Rev Drug Discov 2020;19(05):333-352

75 Larsson M, Rayzman V, Nolte MW, et al. A factor XIla inhibitory antibody provides thromboprotection in extracorporeal circulation without increasing bleeding risk. Sci Transl Med 2014;6 (222):222ra17

76 Matafonov A, Leung PY, Gailani AE, et al. Factor XII inhibition reduces thrombus formation in a primate thrombosis model. Blood 2014;123(11):1739-1746

77 Yau JW, Liao P, Fredenburgh JC, et al. Selective depletion of factor $\mathrm{XI}$ or factor XII with antisense oligonucleotides attenuates catheter thrombosis in rabbits. Blood 2014;123(13):2102-2107

78 David T, Kim YC, Ely LK, et al. Factor XIa-specific IgG and a reversal agent to probe factor XI function in thrombosis and hemostasis. Sci Transl Med 2016;8(353):353ra112

79 Büller HR, Bethune C, Bhanot S, et al; FXI-ASO TKA Investigators. Factor XI antisense oligonucleotide for prevention of venous thrombosis. N Engl J Med 2015;372(03):232-240

80 Weitz JI, Bauersachs R, Becker B, et al. Effect of osocimab in preventing venous thromboembolism among patients undergoing knee arthroplasty: the FOXTROT randomized clinical trial. JAMA 2020;323(02):130-139

81 Combes A, Hajage D, Capellier G, et al; EOLIA Trial Group, REVA, and ECMONet. Extracorporeal membrane oxygenation for severe acute respiratory distress syndrome. N Engl J Med 2018;378(21): 1965-1975

'platelet function' OR 'multiplate' OR 'MEA' OR 'thrombin generation' OR 'endogenous thrombin potential' OR 'platelet aggregation' OR 'light transmittance aggregometry' OR 'PFA' OR 'VerifyNow' OR ‘Global Thrombosis Test' OR 'T-TAS' OR ‘GDF15' OR 'Biomarkers of bleeding' OR 'Biomarkers of thrombosis') in any combination. Articles were filtered based on title and abstract, and reference lists were traced to identify additional articles of relevance. We included case series, observational or interventional studies and review articles, but excluded isolated case reports. There were no language restrictions. 